\title{
NASCIS 2 : prévention des lésions secondaires dans les traumatismes de la moelle épinière
}

Peut-on faire quelque chose pour limiter les séquelles sensitivo-motrices des traumatismes de la moelle épinière? Cette question restait pratiquement sans réponse il y a encore quelques années. Comme pour toutes les destructions neuronales dans le système nerveux central du mammifère adulte, l'absence de prolifération neuronale et l'apparente incapacité des neurones restants à regénérer semblaient interdire toute récupération. L'étude expérimentale des concussions spinales chez l'animal a toutefois ouvert une porte à la thérapeutique en démontrant que les lésions neuronales n'étaient pas toutes directement liées à l'atteinte primaire. A la suite du choc, et pendant une période de plusieurs heures ou jours, des neurones préservés dans un premier temps dégénèrent secondairement.

Cette atteinte secondaire est, de façon hypothétique, associée à des altérations tant du fonctionnement des cellules nerveuses elles-mêmes que de leur environnement cellulaire et biochimique. D'une part, il existe localement une augmentation des concentrations de glutamate et d'aspartate, ces neurotransmetteurs excitateurs devenant alors neurotoxiques (activité excitotoxique) [1]. Ils accroissent l'entrée d'ions calcium dans les neurones et provoquent ainsi leur mort. Des traitements fondés sur des bloqueurs des canaux laissant passer les ions calciques (antagonistes calciques ou antagonistes des canaux NMDA) sont actuellement à l'étude. D'autre part, l'ouverture de la barrière hémato-encéphalique et la réaction inflammatoire s'accompagnent de la libération de substances éventuellement toxiques pour les neurones et les cellules gliales ; parallèlement, la dégradation des lipides membranaires au site de lésion provoque l'apparition de radicaux libres oxygénés neurotoxiques ; il existe, enfin, une réduction tardive du flux sanguin qui peut provoquer une ischémie secondaire relative dans des territoires non atteints jusque-là [2]. C'est contre cet aspect environnemental que tentent d'intervenir les équipes regroupées, aux États-Unis, dans les essais cliniques multicentriques appelés NASCIS (National acute spinal cord injury study). L'agent expérimenté est un corticostéroïde, la méthyl prednisolone (MPSS), dont on a observé chez l'animal que, en plus de son activité anti-inflammatoire, il s'opposait à la dégradation oxygénée des lipides membranaires et, par la même occasion, à la réduction du flux sanguin [3].

NASCIS 1 , interrompu il y a 5 ans, avait donné des résultats décevants. Fondé sur l'injection de $1000 \mathrm{mg}$ de MPSS soit en un bolus soit à raison de $100 \mathrm{mg}$ par jour pendant 10 jours après le traumatisme, il n'avait pas permis d'affirmer un effet de l'un ou l'autre des traitements. NASCIS 2 fut entrepris immédiatement après, sur la base de nouvelles données expérimentales indiquant que des doses beaucoup plus fortes étaient nécessaires, chez l'animal, pour obtenir une diminution significative des séquelles. C'est le résultat de cette étude que vient de publier le New England Journal of Medicine [4].

Pour NASCIS 2, 487 patients ont été inclus à la suite de traumatismes spinaux aigus. Suivant un protocole aléatoire en double-insu, les patients ont reçu soit de la MPSS $(\mathrm{n}=162)$, soit de la naloxone $(n=154)$, soit un placebo $(\mathrm{n}=171)$. L'antagoniste des récepteurs opioïdes a été introduit dans l'essai à la suite de données expérimentales indiquant son effica- cité éventuelle mais NASCIS 2 n'a pas permis de mettre celle-ci en évidence. En ce qui concerne la MPSS, elle était injectée suivant un protocole précis, tout d'abord en un bolus de $30 \mathrm{mg} / \mathrm{kg}$ puis à la dose de $5,4 \mathrm{mg} / \mathrm{kg}$ chaque heure pendant 23 heures, soit un total de $154,2 \mathrm{mg} / \mathrm{kg}$ sur 24 heures. Au total pour un poids de $60 \mathrm{~kg}$, le patient recevait donc en 24 heures 10 fois plus de MPSS qu'au cours de NASCIS 1.

Les résultats obtenus après 6 semaines et 6 mois semblent indiquer un réel effet du traitement (Tableau I). En utilisant des échelles de fonction motrice, de sensibilité à la douleur (piqûre) et de sensibilité tactile, les chiffres obtenus montrent que les patients classés d'abord comme paraplégiques avec perte totale de sensibilité remontent plus sur les trois échelles s'ils ont reçu la MPSS que s'ils ont reçu le placebo. Les résultats sont souvent à la limite de la signification statistique mais globalement du bon côté. En revanche, pour les patients classés comme paraplégiques avec perte de sensibilité partielle, les résultats ne sont pas significatifs. Un autre résultat important de l'étude est la démonstration de l'effet de la MPSS sur les étapes les plus précoces des phénomènes dégénératifs secondaires. En divisant les patients en deux groupes, suivant qu'ils avaient reçu leur première injection à plus ou moins de 8 heures après le traumatisme, les auteurs ont en effet observé que la MPSS perdait son efficacité au cours du temps.

La MPSS apparaît donc comme un traitement préventif potentiellement efficace contre les lésions secondaires des traumatismes spinaux. Une nouvelle étude NASCIS 3 vient d'être entreprise, dans laquelle la durée du 


\begin{tabular}{|l|c|c|c|c|}
\hline \multicolumn{4}{|c|}{ Tableau I } \\
\hline & \multicolumn{2}{|c|}{6 semaines } & \multicolumn{2}{c|}{6 mois } \\
\hline & MPSS & placebo & MPSS & $\begin{array}{c}\text { placebo } \\
\text { (n }=44)\end{array}$ \\
\hline Moteur & $(n=47)$ & $(n=46)$ & $(n=45)$ & $(n)$ \\
Piqûre & $6,2^{* *}$ & 1,3 & $10,5^{* *}$ & 4,2 \\
Tact & 5,9 & 2,2 & $9,4^{*}$ & 4,0 \\
\hline
\end{tabular}

Amélioration des scores sur les échelles motrices, de sensibilité à la piqûre et au tact, chez les paraplégiques avec perte de sensibilité totale, à 6 semaines et à 6 mois après traitement par la MPSS ou par le placebo. * :P $\leqslant 0,05 ; * *: P \leqslant 0,02$.

traitement est prolongée à 48 heures, à la suite de données expérimentales récentes. Il convient, cependant, de rester prudent face à ces résultats. D'abord, parce qu'il est très difficile d'affirmer qu'une récupération de fonction de quelques points (sur une échelle de 70 à 90 ) correspond effectivement à l'action de la drogue. Le nombre de patients inclus dans NASCIS 2 est impressionnant mais la significativité statistique faible, malgré tout, indique pour le moins que l'efficacité du traitement est très inhomogène. Ensuite, et surtout, le tuellement en France comme aux États-Unis car, ainsi que le soulignent les auteurs une augmentation de quelques points d'un score de récupération peut, néanmoins, faire la différence pour le malade entre la dépendance totale et l'autonomie partielle.

M.P. traitement à la MPSS peut ne pas être bénin. Il a existé une augmentation significative du nombre d'infections chez les malades traités dans NASCIS 2, et le nombre de saignements gastriques a été assez élevé quoiqu'il n'ait pas atteint le seuil de signification statistique. Ces réserves ne doivent pas jeter le doute sur l'intérêt potentiel de la drogue mais indiquent que l'on ne peut encore passer à une phase supérieure de l'essai thérapeutique. L'expérimentation clinique doit certainement se poursuivre, et se développer, éven-
1. Meldrum B, Garthwaite J. Excitatory amino acid neurotoxicity and neurodegenerative disease. Trends Pharmacol Sci $1990 ; 11$ : 379-87.

2. Collins WF, Piepmeier J, Ogle F. The spinal cord injury problem : a review. Central Nervous System Trauma 1986 ; 3 : 317-31.

3. Means ED, Anderson DK, Water TR, Kalaf F. Effects of methylprednisolone in compression trauma to the feline spinal cord. $J$ Neurosurg 1981; 55 : 106-13.

4. Bracken MB, Shepard MJ, Collins WF, et al. A randomized, controlled trial of methylprednisolone or naloxone in the treatment of acute spinal-cord injury. $N$ Engl J Med 1990 ; 322 : 1405-11.
Souris transgéniques et hypotension artérielle. Steinhelper $e t$ al. [1], à Cold Spring Harbor (NY, USA), ont créé une lignée de souris qui portent dans leur génome un transgène comprenant les séquences 5' régulatrices du promoteur de la transthyrétine (promoteur actif dans le tissu hépatique) et des séquences d'ADN de souris contenant les 3 exons du gène codant pour le facteur natriurétique auriculaire (ANF). Les souris transgéniques produisent de grandes quantités d'ANF ; le site de synthèse est le foie ; les taux circulants d'ANF sont très élevés, mais seuls $33 \%$ environ représentent de l'ANF mature; le reste correspond à une substance de plus haut poids moléculaire, peut-être le précurseur de l'ANF, le pro-ANF (qui, normalement, est converti en ANF dans les cellules myocardiques et donc ne circule pas). Cela pourrait s'expliquer par la capacité limitée de l'hépatocyte à effectuer cette conversion. Fait remarquable, les souris porteuses du transgène ont une pression artérielle qui est de 25 à $30 \mathrm{mmHg}$ plus basse que celle de souris non transgéniques de même lignée. L'hypotension est observée quel que soit le site d'intégration du transgène (dans une lignée sur quatre, la transmission se fait selon le mode lié au sexe), et quel que soit l'âge des animaux. L'excrétion urinaire d'eau, de sodium et de potassium est identique chez les souris transgéniques et les souris témoins. L'hypotension artérielle pourrait être due aux effets extrarénaux de l'ANF ou de son précurseur. Néanmoins l'hypotension artérielle, entraînant une réduction de la pression de perfusion rénale, pourrait avoir masqué l'action rénale propre de l'ANF ; le pro-ANF, qui représente la forme circulante principale chez les souris transgéniques, pour rait également être insuffisamment converti en ANF et ne pas exercer d'effets rénaux. L'étude des animaux transgéniques devrait apporter des informations intéressantes sur le rôle de certaines hormones et de certains médiateurs dans la régulation cardiovasculaire, chez des animaux soumis très tôt dans le développement à leur influence, et cela de façon prolongée. L'intérêt du travail de Mullins et al. à Heidelberg $\left(\mathrm{m} / \mathrm{s} n^{\circ} 8\right.$, vol. $\left.6, p .826\right)$ et de celui de Steinhelper et al. est bien souligné dans l'éditorial de Gardner [2].

[1. Steinhelper ME, et al. Hypertension $1990 ; 16: 301-7$.]

[2. Gardner DG. Hypertension 1990 ; $16: 308-10$.] 